\title{
Adaptive Co-Channel Interference Suppression Technique for Multi-User MIMO MC DS/CDMA Systems
}

\author{
Prabagarane Nagaradjane ${ }^{1}$, Arvind Sai Sarathi Vasan ${ }^{2}$, \\ Lakshmi Krishnan ${ }^{2}$, Anand Venkataswamy ${ }^{1}$ \\ ${ }^{l}$ Department of Electronics and Communication Engineering, SSN College of Engineering, SSN Institutions, Chennai, India \\ ${ }^{2}$ Department of Electrical and Computer Engineering, University of Maryland, College Park, Maryland, USA \\ E-mail:prabagaranen@ssn.edu.in, \{arvind88,lakshmik\}@umd.edu,anandv267@gmail.com \\ Received September 2, 2009; revised October 10, 2009; accepted November 16, 2009
}

\begin{abstract}
In this paper, an adaptive co-channel interference suppression technique for multi-user MIMO MC DS/CDMA system is envisaged. MC DS/CDMA offers many advantages like flexibility, robustness, low PAPR and spectral efficiency. In spite of these advantages, performance of MC DS/CDMA system is greatly impaired by interference. Common interferences, which degrade the performance of the system, are MAI and CCI. Mitigating these interferences can directly increase the capacity of the system. In this work, an adaptive co-channel interference suppression technique based on single-stage and two-stage MMSE IC is considered for multi-user MIMO MC DS/CDMA system. Simulation results show that, at low SNR two-stage MMSE IC outperforms single-stage, while at high SNR, single-stage provides better BER performance. Based on this, a selection criterion has been propounded for improved system performance as a whole in interference limited environment. Also, adaptive selection criterion resulted in better error performance.
\end{abstract}

Keywords: CCI, MIMO, MC DS/CDMA, MMSE IC, ML Decoding

\section{Introduction}

Multi-carrier transmission has recently gained enormous attention for providing high data rate communications on both forward and reverse channels. Multi-carrier transmission is realized through OFDM.OFDM performs well in frequency selective channels [1]. One of the most promising single carrier transmission schemes is CDMA, which is robust to noise [2]. Also in the recent past, multiinput multi-output (MIMO) has proved to provide very high capacity without any increase in bandwidth or power [3]. Combining these techniques leads to MIMO MC DS/CDMA system, which is expected to meet the demands of future broadband $(4 \mathrm{G})$ wireless wide-area networks. Though MIMO MC DS/CDMA possesses many advantages, it still suffers from the traditional impairments of conventional CDMA systems like MAI and CCI [4-6]. Of late, myriad research concentration has been on proposing techniques for mitigating MAI (Multiple Access Interference) and multi-path, but very little work has been carried out on CCI (Co-Channel Interference) combating techniques. In this paper, we investigate the performance of single-stage and two-stage CCI cancellation techniques for a MIMO MC DS/CDMA system.
MIMO is realized by employing space-time block codes. At the receiver, we have employed two-stage MMSE (Minimum Mean Square Error) IC (Interference Cancellation) with ML (Maximum Likelihood) decoding [4]. We have considered a multi-user MIMO MC DS/CDMA system, with $k$ asynchronous co-channel users in the uplink. Each of the $k$ asynchronous co-channel users is equipped with $N_{\mathrm{T}}$ transmit antennas and they communicate to a single base station, equipped with $N_{\mathrm{R}}$ receive antennas. In this multi-user environment, $k \times N_{\mathrm{T}}$ interfering signals will be arriving at the base station. Conventional interference mitigation technique from $k-1$ cochannel users requires $N_{\mathrm{T}} \mathrm{x}((k-1)+1)$ receive antennas, so as to suppress the co-channel interferences. But by employing STBC the same can be achieved by using $N_{\mathrm{R}}$ receive antennas such that $N_{\mathrm{R}} \geq k$, which exploits the spatial and temporal structure. Also in this work we consider two co-channel users, each equipped with two transmit antennas and the base station equipped with two receive antennas. The performance of MMSE IC and ML decoding algorithm over MC DS/CDMA system with each MC DS/CDMA transmitter in turn carrying multiuser data is assayed for both downlink and uplink. The paper is organized as follows: Section 2 introduces the 
system model we have considered throughout this work, Section 3 describes the improved MMSE IC with ML decoding algorithm, Section 4 describes the two-stage MMSE IC for MIMO MC DS/CDMA system, Section 5 expounds the performance results and Section 6 provides the conclusions.

\section{System Models}

Here the system model that we have considered comprises two asynchronous co-channel users in a MIMO MC DS/CDMA system in which each transmitting terminal is a MC DS/CDMA transmitter with MIMO support realized through Space Time Block Codes (spatial diversity). The two co-channel users communicate with two receive antennas at the receiver, which performs interference cancellation and then detects the transmitted signal of each user. Figure 1 shows the MIMO MC DS/ CDMA transmitter. The user data at each transmitting terminal after appropriate constellation mapping is multiplied with a spreading code and the spread symbols of each data are multi-carrier modulated. Then the sum of all the carriers of the $k$ th user composes the output of the $k$ th user signal $S_{k}(t)$. The total output $S(t)$ is the sum of all the user signals. After HPA (high power amplification), the final signal is transmitted. The transmitted signal corresponding to the $n$th data symbol of the $k$ th user is

$$
S_{n}^{k}(t)=\sum_{m=0}^{M-1} \sum_{q=0}^{Q-1} d_{m}^{k} C_{m, q}^{k} p_{m}\left(t-q T_{c}-n T\right) e^{j 2 \pi f_{m} t}
$$

where, $k$ is the user number, $m$ is the carrier number, $q$ is the chip number, $T_{c}$ is chip duration and $T$ is symbol duration and equals to $Q T_{c}$. $Q$ is the length of user specific spreading code. $d_{m}{ }^{k}$ is the data of $m$ th sub-carrier and $k$ th user, $c_{m, q}{ }^{k}$ is the $q$ th spreading code of $m$ th carrier of $k$ th user, $p_{m}(t)$ is Root raised cosine pulse of $m$ th carrier and $f_{m}$ is the $m$ th carrier frequency. When the total number of users is $K$, the total transmitted signal corresponding to the $n$th data symbol is

$$
S_{n}(t)=\sum_{k=0}^{K-1} \sum_{m=0}^{M} \sum_{q=0}^{Q-1} d{ }_{m}^{k} C_{m, q}^{k} e^{j 2 \pi m \Delta f t}
$$

This modulated stream is then passed through a STBC encoder which groups the symbols according to a specific STBC pattern (G2) and then transmits the symbols through multiple transmit antennas. The received vector at the first receive antenna for the transmitted symbols $\boldsymbol{d}_{1}$ and $\boldsymbol{d}_{\mathbf{2}}$ is expressed as

$$
\left[\begin{array}{l}
r_{1} \\
r_{2}
\end{array}\right]=\left[\begin{array}{cc}
d_{1} & d_{2} \\
-d_{2}^{*} & d_{1}^{*}
\end{array}\right]\left[\begin{array}{l}
h_{11} \\
h_{21}
\end{array}\right]+\left[\begin{array}{l}
n_{11} \\
n_{21}
\end{array}\right]
$$

where, $\boldsymbol{h}_{11}$ and $\boldsymbol{h}_{21}$ denotes the channel fading and $\boldsymbol{n}_{11}$ and $\boldsymbol{n}_{21}$ represents the additive white Gaussian noise

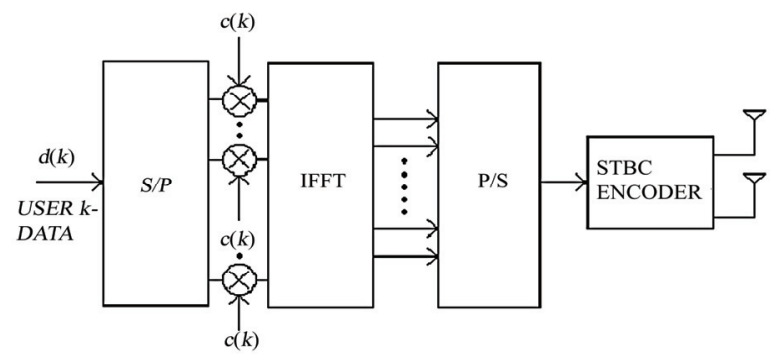

Figure 1. MIMO MC DS/CDMA transmitter.

(AWGN). Let $\boldsymbol{u}_{1}, \boldsymbol{u}_{2}$ and $\boldsymbol{v}_{1}, \boldsymbol{v}_{2}$ represent the symbols transmitted over two consecutive symbol durations by the corresponding two co-channel asynchronous users. As shown in Figure 2, let $\boldsymbol{h}_{11}, \boldsymbol{h}_{21}, \boldsymbol{g}_{11}$ and $\boldsymbol{g}_{21}$ represent the channel fading between all the transmit antennas and the first receive antenna at the base station respectively and $\boldsymbol{h}_{12}, \boldsymbol{h}_{22}, \boldsymbol{g}_{12}$ and $\boldsymbol{g}_{22}$ represent the channel fading between all the transmit antennas and the second receive antenna at the base station respectively. Now, the received vector at the receive antenna Rx1 over the two symbol time period is expressed as

$$
\begin{aligned}
& r_{11}=h_{11} u_{1}+h_{21} u_{2}+g_{11} v_{1}+g_{21} v_{2}+n_{11} \\
& r_{12}=-h_{11} u_{2}^{*}+h_{21} u_{1}^{*}-g_{11} v_{2}^{*}+g_{21} v_{1}^{*}+n_{12}
\end{aligned}
$$

The received vector at the receiver antenna $\mathrm{Rx} 2$ is given by

$$
\begin{gathered}
r_{21}=h_{12} u_{1}+h_{22} u_{2}+g_{12} v_{1}+g_{22} v_{2}+n_{21} \\
r_{22}=-h_{12} u_{2}^{*}+h_{22} u_{1}^{*}-g_{12} v_{2}^{*}+g_{22} v_{1}^{*}+n_{22}
\end{gathered}
$$

\section{Improved MMSE IC ML Decoding}

The overall received signal from each of the receive antenna is expressed as

$$
r=\left[\begin{array}{ll}
R_{1}^{T} & R_{2}^{T}
\end{array}\right]^{T}
$$

The channel fading coefficient between each transmitter and the receive antenna is rearranged for each cochannel user to form a generic channel matrix. For detecting the first co-channel user data, the corresponding channel matrix is represented as

$$
H=\left[\begin{array}{ll}
H_{1} & G_{1} \\
H_{2} & G_{2}
\end{array}\right]
$$

and for the second user, it is given by

$$
\widetilde{H}=\left[\begin{array}{ll}
G_{1} & H_{1} \\
G_{2} & H_{2}
\end{array}\right]
$$

The MMSE weight matrix is calculated based on the generic channel matrix and the weight matrix value dif- 


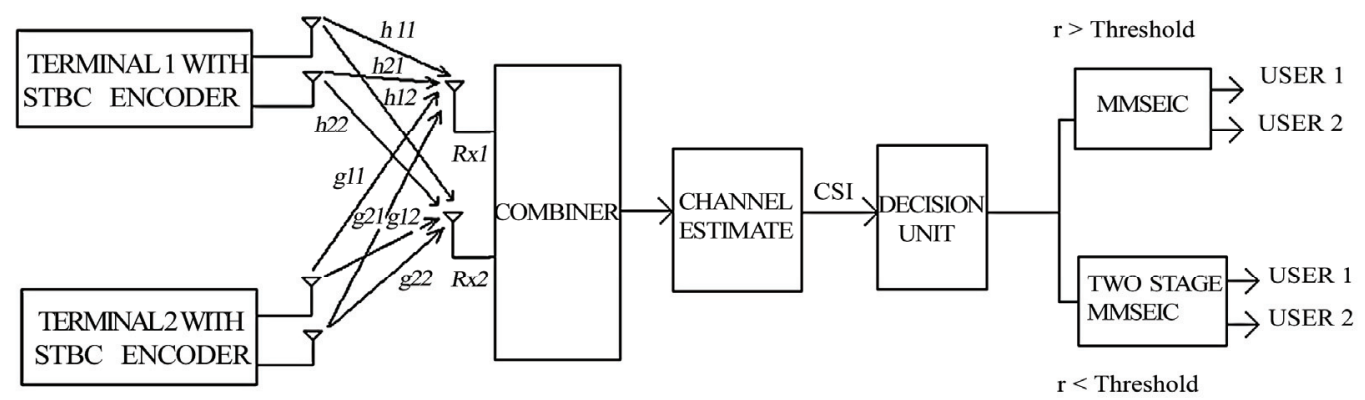

Figure 2. MIMO MC DS/CDMA with two co-channel asynchronous users and adaptive base station receiver system model.

fers for each co-channel user. For each of the two users considered here in this work, the MMSE weight matrix is computed from the expression $[4,5]$

$$
M=H H^{H}+\sigma^{2} I_{4} \text { and } \tilde{M}=\tilde{H} \widetilde{H}^{H}+\sigma^{2} I_{4}
$$

where, $\boldsymbol{M}$ is the weight matrix for the first user and $\tilde{M}$ is the weight matrix for the second user. $H^{\mathrm{H}}$ represent the Hermitian transpose of the channel matrix and $\sigma^{2}$ is the noise variance. To suppress the interferences from other co-channel users, the inverse of the weight matrix is multiplied with the columns of the channel matrix that represents the channel fading for a particular cochannel user i.e.

$$
w_{1}=M^{-1} h_{1} \text { and } \widetilde{w}_{1}=\tilde{M}^{-1} h_{1}
$$

where,

$$
\begin{aligned}
& \boldsymbol{h}_{1}=\text { first column of } H \text { or } \widetilde{H} \\
& \boldsymbol{h}_{2}=\text { second column of } H \text { or } \widetilde{H}
\end{aligned}
$$

The MMSE Interference cancellation receiver suppresses both co-channel interferences and noise components, which means that the mean square error or variance between the transmitted symbols and the estimate is reduced. The maximum likelihood (ML) detection is used to detect the transmitted symbols for the corresponding user. The ML decoding estimates the symbols by determining the minimum Euclidian distance of all possible transmitted symbols from the received constellation [4], given by

$$
\hat{U}=\underset{\hat{u} \in U}{\arg \min }\left\{\left\|w_{1}^{H} r-\hat{u}_{1}\right\|^{2}+\left\|w_{2}^{H} r-\hat{u}_{2}\right\|^{2}\right\}
$$

and

$$
\hat{V}=\underset{\hat{v} \in V}{\arg \min }\left\{\left\|\widetilde{w}_{1}^{H} r-\hat{v}_{1}\right\|^{2}+\left\|\widetilde{w}_{2}^{H} r-\hat{v}_{2}\right\|^{2}\right\}
$$

where, $\hat{U}$ and $\hat{V}$ represent the two co-channel users estimated data. $\hat{u}_{1}, \hat{u}_{2}, \hat{v}_{1}$ and $\hat{v}_{2}$ takes all possible values of the users signal constellation. The reliability of the decoded signals are computed by

$$
\gamma_{c 0}=\left\|w_{1}^{H} r-\hat{u}_{1}\right\|^{2}+\left\|w_{2}^{H} r-\hat{u}_{2}\right\|^{2}
$$

$$
\gamma_{s 1}=\left\|w_{1}^{H} r-\hat{v}_{1}\right\|^{2}+\left\|w_{2}^{H} r-\hat{v}_{2}\right\|^{2}
$$

\section{Two Stage Interference Cancellation}

The two stage interference cancellation proceeds with first decoding the data from the two terminals. Then, assuming each decoded value is correct, the data corresponding to the other user is estimated using Maximum likelihood estimation. i.e. first assuming the first terminal decoded data is correct based on single stage MMSE IC, the second user data is estimated from the decoded data. This is carried out by first calculating $\mathbf{x}_{\mathbf{1}}$ and $\mathbf{x}_{\mathbf{2}}$.

$$
x_{1}=R_{1}-H_{1} \cdot \hat{c}_{0} \text { and } x_{2}=R_{2}-H_{2} \cdot \hat{\mathcal{c}}_{0}
$$

where, $\hat{c}_{0}$ is the data decoded, corresponding to the first user. The second user data is estimated from the first user decoded data by using the maximum likelihood estimation given by

$$
\hat{s}_{0}=\underset{\hat{s}_{0} \in S}{\arg \min }\left\{\left\|x_{1}-G_{1} s\right\|^{2}+\left\|x_{2}-G_{2} s\right\|^{2}\right\}
$$

where, $\boldsymbol{S}$ takes all possible values in the signal constellation. The reliability corresponding to the estimated data is given by the expression

$$
\gamma_{s 0}=\left\|x_{1}-G_{1} \hat{s}_{0}\right\|^{2}+\left\|x_{2}-G_{2} \hat{s}_{0}\right\|^{2}
$$

Similarly, the first user data based on single stage MMSE IC is estimated by computing $\mathbf{y}_{\mathbf{1}}$ and $\mathbf{y}_{2}$.

$$
y_{1}=R_{1}-G_{1} . \hat{s}_{1} \text { and } y_{2}=R_{2}-G_{2} \cdot \hat{s}_{1}
$$

where, $\hat{s}_{1}$ is the data decoded corresponding to the second user. The first user data is estimated from the second user decoded data by using the expression [4]

$$
\hat{c}_{1}=\underset{\hat{c}_{1} \in C}{\arg \min }\left\{\left\|y_{1}-H_{1} c\right\|^{2}+\left\|y_{2}-H_{2} c\right\|^{2}\right\}
$$

The corresponding value of reliability for the estimated data is

$$
\gamma_{c 1}=\left\|y_{1}-H_{1} \hat{c}_{1}\right\|^{2}+\left\|y_{2}-H_{2} \hat{c}_{1}\right\|^{2}
$$


The receiver computes the overall reliability for the two users i.e. $\gamma_{0}=\gamma_{c 0}+\gamma_{s 0}$ and $\gamma_{1}=\gamma_{c 1}+\gamma_{s 1}$. The decision is made on the sets of symbols computed by comparing the two reliabilities. The comparison is made as [4]

$$
\begin{gathered}
\text { if }\left(\gamma_{0}<\gamma_{1}\right) \\
(\hat{c}, \hat{s})=\left(\hat{c}_{0}, \hat{s}_{0}\right) \\
\text { else } \\
(\hat{c}, \hat{s})=\left(\hat{c}_{1}, \hat{s}_{1}\right)
\end{gathered}
$$

The system illustrated in Figure 2, consists of a receiver with a switch between a single and double stage MMSE IC unit, CSI (Channel State Information) and a decision unit. When the channel is slowly varying, the receiver detects the symbols based on single stage technique. When the channel variation is rapid, two stage MMSE IC is employed to detect the symbols. At present, perfect channel knowledge is assumed at the receiver. The entire detection takes place based on the instantaneous SNR available at the receiver.

\section{Performance Analysis}

In this section, we present the performance of adaptive co-channel suppression technique for a multi-user MIMO MC DS/CDMA system. The simulation results for the single stage and two stage interference cancellation techniques are shown in Figures 3 and 4. The channel model considered is quasi-static Rayleigh fading channel, which is built on the classical understanding of Doppler shift and delay spread. The modulation scheme employed is BPSK, as it provides the best system throughput for MIMO realization based on STBC. The number of channel realizations considered for uplink and downlink is 5000 and 10000 respectively for each value for SNR. Table 1 summarizes the simulation parameters.

Simulation results divulge that, at low SNR value, two stage interference cancellation techniques perform well whereas at high SNR value single stage MMSE IC with ML decoding provides better BER performance. Hence, a trade off can be made in selecting the interference cancellation techniques at the receiver when the SNR dwindles. This can result in better performance of MIMO MC DS/CDMA system in an interference limited environment as switching of IC can be made in an adaptive manner.

Figure 3 shows the uplink performance of MIMO MC DS/CDMA system with single stage IC, two stage IC and adaptive IC for two co-channel users. Each user data is spread by a spreading factor of 32 . Here in each MC DS CDMA system one user is accommodated. It can be discerned that adaptive IC outperforms both single stage

\begin{tabular}{|c|c|c|}
\hline \multicolumn{2}{|c|}{ PARAMETERS } & VALUES \\
\hline \multicolumn{2}{|c|}{ Spreading code } & Walsh Hadamard \\
\hline \multirow{2}{*}{$\begin{array}{l}\text { Number of } \\
\text { channel reali- } \\
\text { zations }\end{array}$} & Uplink & 5000 \\
\hline & Downlink & 10000 \\
\hline \multicolumn{2}{|c|}{ FFT Size } & 128 \\
\hline \multicolumn{2}{|c|}{ Cyclic Prefix } & $1 / 8$ \\
\hline \multicolumn{2}{|c|}{ Spreading Factor } & 16 or 32 \\
\hline \multicolumn{2}{|c|}{ Data Modulation } & BPSK \\
\hline \multicolumn{2}{|c|}{ Channel Model } & Rayleigh \\
\hline \multicolumn{2}{|c|}{ Number of Transmit antennas } & 2 \\
\hline \multicolumn{2}{|c|}{ Number of Receive antennas } & 2 \\
\hline
\end{tabular}
and double stage. The same performance can also be realized over the downlink channel. Figure 4 elucidates
Table 1. Simulation parameters.

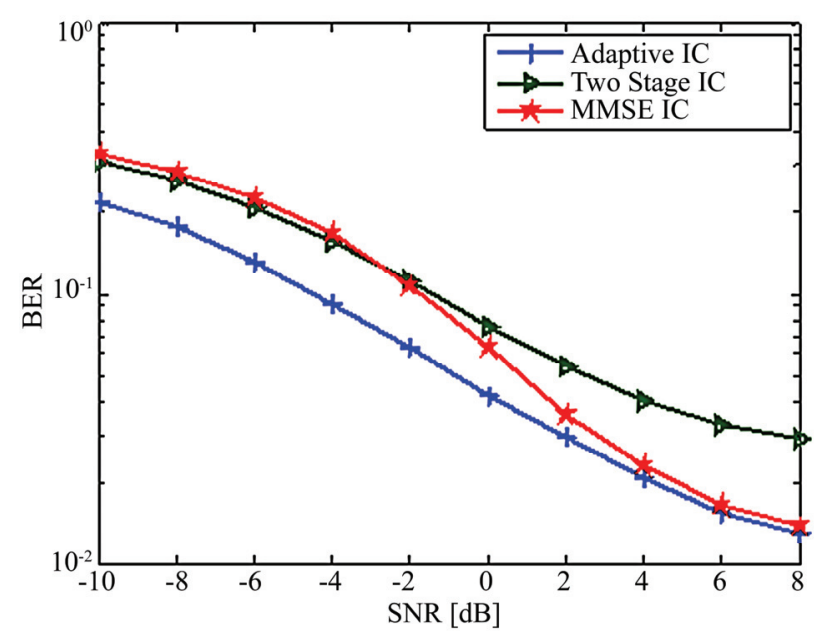

Figure 3. Performance of adaptive co-channel interference scheme for 2 co-channel users over an uplink communication channel for MIMO MC DS/CDMA system.

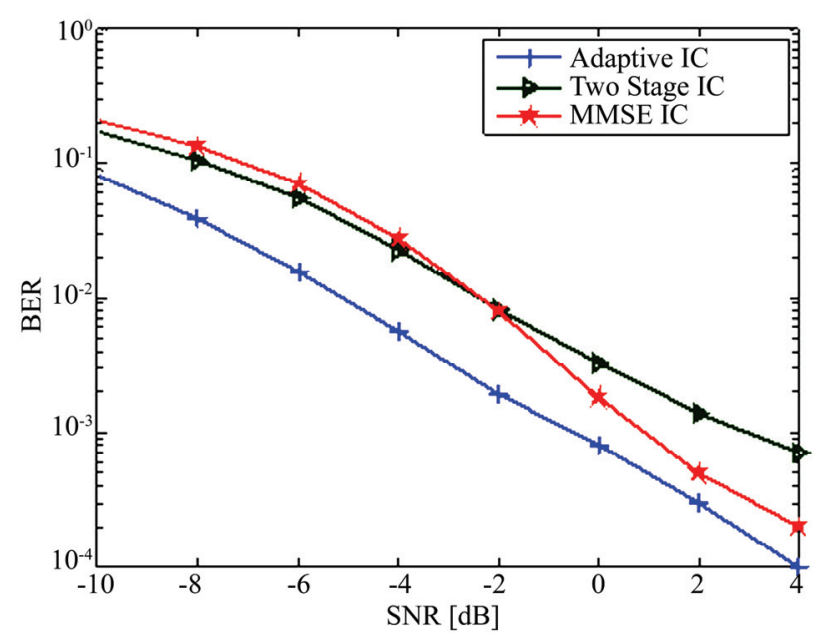

Figure 4. Performance of adaptive co-channel interference scheme for 4 co-channel users over downlink communication channel for MIMO MC DS/CDMA system. 
the performance of the same system with four co-channel users, with each user spread by a spreading factor of 16 over downlink communication channel. Here also adaptive switching scheme provides better BER performance.

\section{Conclusions}

In this work, we considered a two stage MMSE co-channel interference cancellation receiver for MIMO MC DS/ CDMA systems. MC DS /CDMA can be realized as a prominent air interface for $4 \mathrm{G}$ Broadband communications; however, capacity of such systems is limited by interference. Mitigating the various interferences can result in confronting the future generation wireless networks needs. In this paper we have analyzed a two stage IC technique for a multi-user environment. Results of our analysis reveal that a trade off could be made in selecting the IC techniques for mitigating CCI. It could be discerned that at low SNR values two stage has resulted in better performance because of its iterative nature while at high SNR values, single stage performs better. Also, it is expounded from our analysis that the adaptive interference cancellation receiver has resulted in better suppression of CCI.

\section{References}

[1] L. Hanzo, L-L. Yang, E-L. Kuna, and K. Yen, "Single and multi-carrier DS-CDMA multi-user detection, space-time spreading, synchronization and standards," IEEE Press, 2003.

[2] M. K. Simon and M. S. Alouini, "BER performance of multi-carrier DS-CDMA systems over generalized fading channels," IEEE International Conference, 1999.

[3] E. Bigieri, R. Calderbank, A. Constantinides, A. Goldsmith, A. Paulraj, and H. Vincent Poor, "MIMO wireless communications," Cambridge University Press, 2007.

[4] Anand. V, Arvind. S, and Lakshmi Krishnan, "Investigations on the performance of MIMO assisted Multi Carrier DS/CDMA system with multi-user detection for 4G mobile communications," Dissertation, SSN Institutions, 2009.

[5] Prabagarane Nagaradjane, Arvind Sai Sarathi Vasan, and Lakshmi Krishnan, "A robust space time co-channel interference mitigation and detection technique for multi-user MIMO multi-carrier DS/ CDMA systems," Proceedings of IEEE International Conference, Wireless Vitae, 2009.

[6] S. Kondo and L. B. Milstein, "Performance of multi-carrier DS CDMA systems", IEEE Transactions on Communications, Vol. 44, No. 2, February 1996. 